\title{
DRP1 links mitochondrial dynamics to the clock
}

The circadian clock coordinates mitochondrial gene expression and bioenergetics, but the mechanisms linking the clock to the mitochondrial network are unknown. New research by Anne Eckert and colleagues shows that the circadian clock controls mitochondrial fission-fusion dynamics through clock-dependant modification of dynamin-related protein 1 (DRP1; also known as DNM1L).

TP16

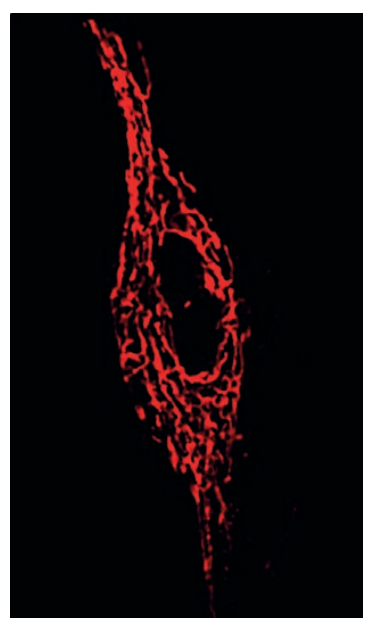

Circadian control of mitochondrial network morphology in serum-shocked human skin fibroblasts at 16 hours (TP16) and 28 hours (TP28). Images courtesy of Karen Schmitt, University of Basel, Switzerland.
The researchers found that mitochondrial oxygen consumption exhibited a 'diurnal' oscillation in which cultured human fibroblasts showed time-of-day-dependent variation in ATP levels. We know that tubular networks formed by mitochondrial fusion upregulate oxidative respiration while mitochondrial fission, which forms smaller fragments, results in the opposite. Eckert and colleagues found that the mitochondrial network existed in distinct morphological states that matched the 'diurnal' oscillation of ATP levels and oxidative phosphorylation. A tubular state occurred during the high oxidative phosphorylation phase (high ATP levels) and a fragmented state when ATP level was low.

DRP1 is an important mediator of mitochondrial fission and phosphorylation of DRP1 on serine 637 promotes mitochondrial elongation. The researchers found that phosphorylated levels of DRP1 showed a 24 hour rhythm that matched the measurements of oxidative phosphorylation and morphological assessments. Suppressing DRP1 activity in vitro and in vivo disrupted the 'diurnal' oscillation of ATP production. Eckert and colleagues found that loss of DRP1 impairs the core circadian clock. Inhibiting DRP1 function in vitro increased the length of the circadian period and repressed the expression of circadian clock genes, including period circadian protein homologue 1 and 2 (Per 1 and Per2, respectively).

"Cell cycle phase directly determines mitochondrial productivity through the control of DRP1 phosphorylation and, with it, mitochondrial morphology," concludes Steven Brown, senior author. "I find it exceptionally elegant that the circadian clock has essentially co-opted this more ancient mechanism to achieve the same global control in time-of-day-dependent fashion via the same control point."

Ivone Leong

ORIGINAL ARTICLE Schmitt, K. et al. Circadian control of DRP1 activity regulates mitochondrial dynamics and bioenergetics. Cell Metab. 27, 1-10 (2018) 


\section{Publisher Correction: DRP1 links mitochondrial dynamics to the clock}

\section{Ivone Leong}

Nature Reviews Endocrinology (2018) https://doi.org/10.1038/nrendo.2018.32

Published online 09 March 2018

In the original version of the published article, the reference was incorrect. The reference should have read 'Schmitt, K. et al. Circadian control of DRP1 activity regulates mitochondrial dynamics and bioenergetics. Cell Metab. https://doi.org/10.1016/j.cmet.2018.01.011 (2018)'.

https://doi.org/10.1038/s41574-018-0071-8 I Published online: 30 July 2018 